\title{
Evaluation of Quality Control Circle Activities in Improving the Delayed Cord Clamp Rate of Newborns Undergoing Vaginal Delivery
}

\author{
Xinke Huang*, Li Zuo, Ruihong Chen, Baohua Qiu, Wenfei Liang \\ Gynaecology and Obstetrics Department, The First Affiliated Hospital of Jinan University, Guangzhou, China \\ Email address: \\ 9299018382@qq.com (Xinke Huang),yi564366593@qq.com (Li Zuo), 184169258@qq.com (Ruihong Chen), \\ 445117898@qq.com (Baohua Qiu),845344955@qq.com (Wenfei Liang) \\ ${ }^{*}$ Corresponding author
}

To cite this article:

Xinke Huang, Li Zuo, Ruihong Chen, Baohua Qiu, Wenfei Liang. Evaluation of Quality Control Circle Activities in Improving the Delayed Cord Clamp Rate of Newborns Undergoing Vaginal Delivery. Journal of Gynecology and Obstetrics. Vol. 7, No. 6, 2019 , pp. 155-160. doi: $10.11648 /$ j.jgo.20190706.11

Received: September 17, 2019; Accepted: September 29, 2019; Published: October 17, 2019

\begin{abstract}
Probing into the Quality Control Circle (QCC) activities in improving the delayed cord clamp rate (DCC) of newborns undergoing vaginal delivery. By setting up QCC team, to investigate and diagnose the problems of DCC rate of newborns undergoing vaginal delivery in our hospital in 2017, therefore, to draw up our new target and the responding measurement. After that, we analyze and discuss the data, and finally filter the valid data. Finally, we compare the two sets of situations and data. According to the investigation of DCC rate undergoing vaginal delivery and the relative Maternal-and-Infant outcome in August to November in 2017, after setting up QCC activities, it shows the DCC rate of newborns undergoing vaginal delivery has an significant increased, from $35.1 \%$ to $82.3 \%$, duration of the Third Stage of Labor, hospitalization rate of Neonatal intensive Care Nursing (NICU), Apgar score, incidence of postpartum hemorrhage, asphyxia neonatorum and hyperbilirubinemia has no statistical sence $(\mathrm{P}>0.05)$. The Quality Control Circle $(\mathrm{QCC})$ activities can effectively improve the delayed cord clamp rate (DCC) of newborns undergoing vaginal delivery, without affecting the incidence of undesirable Maternal-and-Infant outcome. And the quality control circle activities can be fed back and improved through the PDCA cycle to make it more scientific and practical.
\end{abstract}

Keywords: Quality Control Circle (QCC), Newborns, Delayed Cord Clamp (DCC)

\section{Introduction}

Immediate cord ligation of newborns are normal operation in Obstetrics, has been considered it as a positive management at the Third Stage of Labor, in order to prevent or at least to reduce the rate of postpartum hemorrhage and retained placenta. Delayed cord clamp (DCC), also named as late umbilical cord amputation, means to amputate the cord until the newborns have built up their stable respiration and the cord pulsation stopped [1]. A study indicates that delayed cord clamp does not additionally increase the incidence of undesirable Maternal-and-Infant outcome [2]. Besides, compare with immediate cord ligation, delayed cord clamp can increase hemoglobin, hematocrit and serum iron level of newborns, in order to ameliorate their cardiovascular, cerebral, pulmonary system and so as their nervous system development [3]. Quality Control Circle is an effective managing tool to promote the continuing improvement of medical quality, it makes brilliant results in both nursing technique and management [4-6]. In China, the Quality Control Circle is an imperfect technique, that it needs to be adapted to China's medical situation. Nowadays, In addition to obstetrics, other disciplines are beginning to accept the quality control circle, that they use the quality control circle to improve the efficiency and quality of care [20]. Therefore, in 2017, we made QCC activities as new method of delayed cord clamp of newborns undergoing vaginal delivery in our department and we have gotten a good achievement showed as below [7]:

We have chosen 1073 cases of newborns undergoing 
vaginal delivery in our hospital in January to April in 2017, which has 574 males and 499 females, born at $38.95 \pm 0.96^{\text {th }}$ week, birth weight at $3.20 \pm 0.37 \mathrm{~kg}$. Exclusion criteria: 1 . Maternal diseases: blood diseases like anemia, severe perinatal complication like placenta previa, placenta abruption, vasa previa bleeding, multiple pregnancy and hemolysis; 2 . Congenital anomaly of newborn infants like meningocele, congenital heart diseases and alimentary anomaly; 3 . Premature infants. Statistics indicates that two groups of the newborns in general information have no statistics differences $(\mathrm{P}<0.05)$, which possess comparability.

\section{Methods}

\subsection{Set Up QCC Team}

This team includes 8 nurses and 1 chief physician. Matron acts as the team leader who are responsible for planning and leading teammates on completing tasks. Chief physician acts as counselor who are responsible for confirming topics of new missions, providing theoretical guidance and quality control of the team activities. Matron of Gynecology and obstetrics department and matron of maternity wards act as team secretary, who are responsible for coordination and assignment with other departments. Rest of the teammates, including 1 supervisor nurse, 3 senior nurses, 1 obstetric nurse, are responsible for cooperating with the team leaders to implement our fore plans.

\subsection{Topic Confirmation}

We have 8 volunteers who applied for the topic choosing process. We have used the QCC activities matrix table, scoring at four dimensions including importance, emphasis and urgency of nurses and the skills of our teammates. Election score: 5-The highest, 3-Average, 1-The lowest, delayed cord clamp rate of newborns undergoing vaginal delivery came out the first score to be our new topic. Name of Our QCC team was Neonatal-loving Circle, moral of the name is to protect newborns diligently.

\subsection{Formulating New Program}

According to the topic, we made it by using $5 \mathrm{~W} 1 \mathrm{H}$, including drawing up plans, grasping current situation, analyzing key factors, setting goals, analyzing problems, drawing up the countermeasures, implementing and evaluating countermeasures, confirming effect, standardizing, reviewing and improving, etc, and drawing a time gantt chart.

\subsection{Analysis of the Situation}

According to the analysis of 1,073 newborns who were investigated from January to April in 2017 through vaginal delivery, it was found that: the number of delayed cord clamp (DCC) from January to April was 377, and the delayed cord clamp (DCC) rate was $35.1 \%$. The results of the checklist showed that the reasons for the failure of delayed cord clamp (DCC) in such newborns were as follows: obstetric nurse factors (including obstetric nurse lack of knowledge, without standard training, lack of human resources, etc.) with 387 cases, 158 cases of maternal and their families disagred, 53 cases of maternal had intrapartum complications, 98 cases of the doctor factors (the concept had not changed and no medicine indications still urged obstetric nurse to cut navel as soon as possible). See Figure 1 for details. According to the "80/20 decision law", the main reasons for the failure of delayed cord clamp (DCC) in newborns delivered vaginally are the obstetric nurse factors and the disapproval of maternal and their families [8].

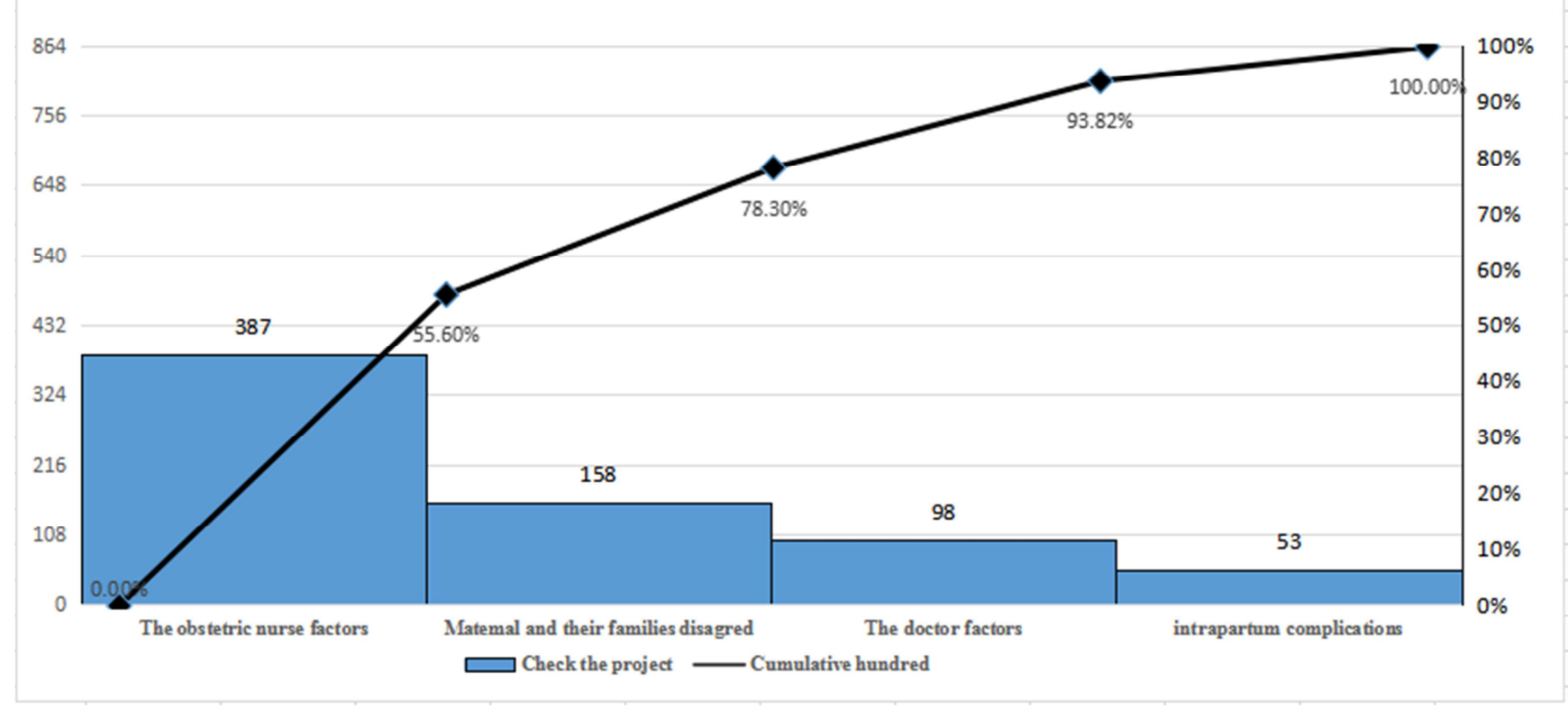

Figure 1. Reasons for failure to perform delayed cord clamp (DCC) Plato. 


\subsection{Goal Setting}

According to the calculation formula of QCC activity target: activity target $=$ present value - (present value $\times$ key improvement $\times$ circle ability), calculated that after implementing QCC activity, the target value of delayed cord clamp (DCC) rate was $75.8 \%$ [9].

\subsection{Analysis of Critical Factors}

The reasons were found from the four aspects of "human, material, legal and environmental", and drawn a fishbone diagram (Figure 2), with a total of 26 terminal factors. After comprehensive consideration of the feasibility and safety of the measures, the main reasons were proved, and the main factors were determined as follows: (1) The doctors and obstetric nurse did not change their concepts and did not have medical indications, but still performed cord clamp immediately after delivery; (2) obstetric nurse lack of standardized training on delayed cord clamp (DCC); (3) No definite indication of delayed cord clamp (DCC); (4) Lack of standardized process of delayed cord clamp (DCC) of newborns after vaginal delivery; (5) Cord blood should be collected from newborns.

\subsection{Formulate and Implement Countermeasures}

\subsubsection{Conduct Standard Training on Delayed Cord Clamp (DCC)}

(1) Add relevant guidance on delayed cord clamp (DCC) to the obstetrics professional learning jointly participated by medical staff. (2) Obstetric nurse were organized to carry out professional learning according to "clinical implementation Suggestions of early neonatal basic health care technology" [10] and the related content of delay cord clamp (DCC) published by the American academy of pediatrics, once a week, a total of two weeks [2], in order to prompt the midwife changing ideas, importance of the delay cord clamp (DCC), and master the delay cord clamp (DCC) related skills.

\subsubsection{The Indications of Delayed Cord Clamp (DCC) Were Clear}

(1) Infant and puerpera are no contraindications of delayed cord clamp (DCC) and complications during delivery. (2) Premature infants score well and have vitality. (3) Puerpera and their families agreed to delay cord clamp (DCC) $[2,10]$. The flowchart of delayed cord clamp (DCC) for newborns of vaginal delivery was developed, and the delayed cord clamp (DCC) was included in the quality control index of the midwifery specialty in 2018 .

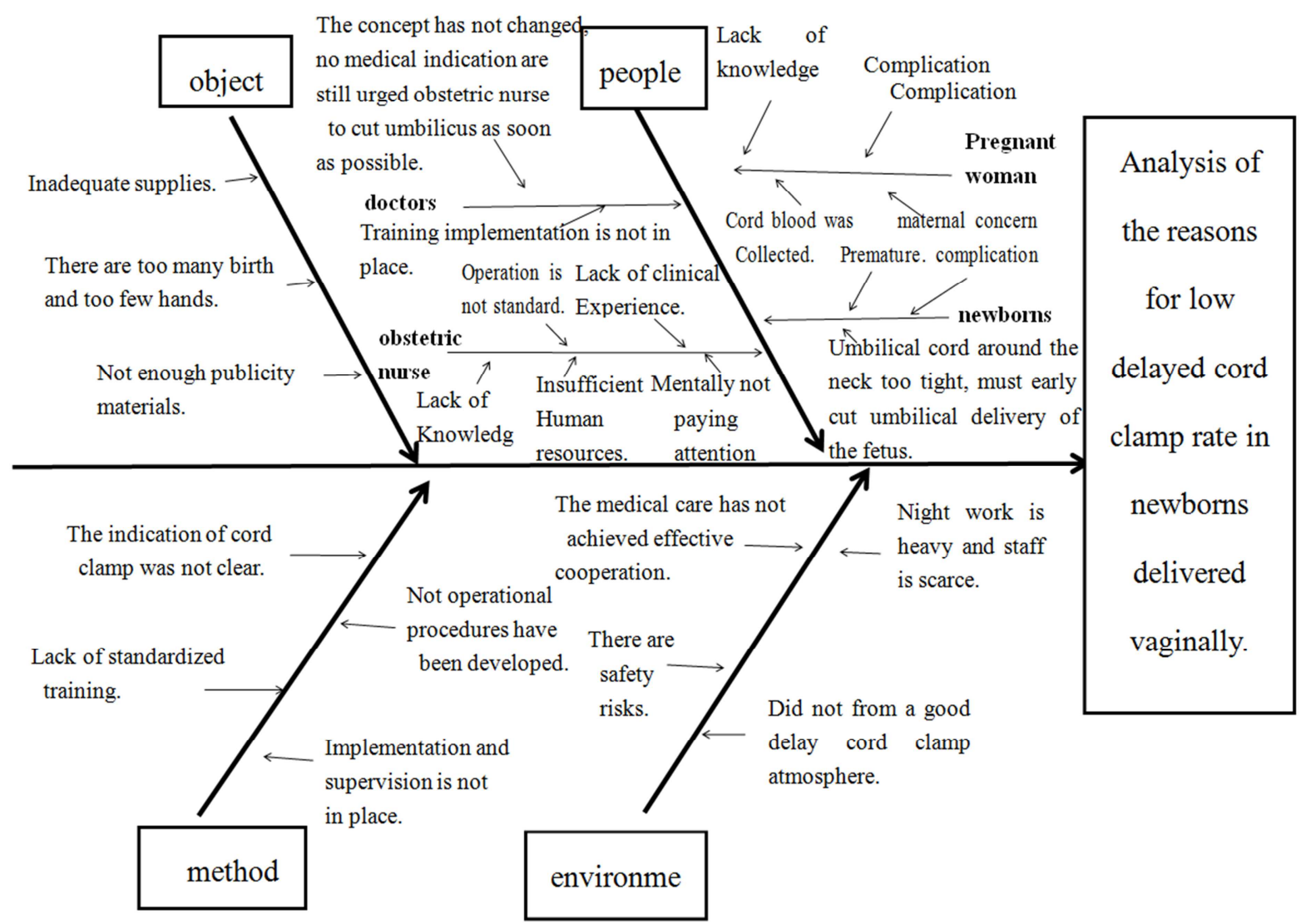

Figure 2. Analysis of the fishbone diagram of delayed cord clamp (DCC) in newborns after vaginal delivery.

\subsubsection{To Strengthen the Education of Perinatal Pregnant Women and Their Families}

The specific ways are as follows: (1) Add delayed cord clamp (DCC) to the school for pregnant women. The school for pregnant women opens every Friday morning, and one class a month is guaranteed to include delayed cord clamp (DCC). (2) 
Antenatal admission education: for pregnant women over 28 weeks of pregnancy, no matter whether they have received education or not, they should inform pregnant women of the relevant knowledge of delayed cord clamp (DCC), which should be taken charge of by the responsible nurse and outpatient education by obstetric nurse; (3) During delivery: obstetric nurse actively solicits opinions from pregnant women and their families, encourages expectant fathers to participate in childbirth, reduces the fear of pregnant women, and informs them of the benefits and importance of delayed umbilical amputation, so that pregnant women and their families cooperate with obstetric nurse to delay cord clamp (DCC) and make them play the role of parents as soon as possible.

\section{Result Confirmation}

\subsection{Comparison of Delayed Cord Clamp (DCC) of Newborn in Vaginal Delivery Before and After the Implementation of QCC}

Before and after the implementation of the activity, the comparison results of delayed cord clamp (DCC) rate of newborn after vaginal delivery were shown in Table 1 . $\mathrm{X}^{2}=, \mathrm{P}<0.001$, the difference was statistically significant. After the implementation of the activity, the delayed cord clamp (DCC) rate of such newborns was significantly improved. The condition of delayed cord clamp (DCC) caused by obstetric nurse factors, maternal and family disagreement and doctor factors was improved, and the difference was statistically significant $(\mathrm{P}<0.05)$.

Table 1. No delayed cord clamp (DCC) before and after the implementation of the quality control circle.

\begin{tabular}{lllll}
\hline & $\begin{array}{l}\text { The number of delayed } \\
\text { cord clamp (DCC) }\end{array}$ & $\begin{array}{l}\text { The obstetric nurse } \\
\text { factors }\end{array}$ & $\begin{array}{l}\text { Matemal and their } \\
\text { families disagreed }\end{array}$ & $\begin{array}{l}\text { Intrapartum } \\
\text { complications }\end{array}$ \\
\hline Before carry out & $377(35.1 \%)$ & 387 & 158 & 53 \\
The doctor factors \\
After carry out & $1053(82.3 \%)$ & 0 & 139 & 43 \\
$P$ & 545.292 & 552.149 & 7.868 & 3.708 \\
$P$ & $P<0.001$ & $P<0.001$ & 0.005 & 0.054 \\
\hline
\end{tabular}

\subsection{Comparison of Related Complications of Parturient and Newborns Before and After the Implementation of QCC}

Table 2 compares the incidence of related complications of parturients and newborns before and after the implementation of the activity. The results showed that there were no statistically significant differences in the duration of third labor, incidence of postpartum hemorrhage, NICU hospitalization rate, incidence of hyperbilirubinemia and incidence of newborns asphyxia $(\mathrm{P}>0.05)$.

Table 2. Complications related to parturients and newborns before and after the implementation of QCC activities.

\begin{tabular}{lllll}
\hline & $\begin{array}{l}\text { the duration of } \\
\text { third labor }(\mathbf{m i n})\end{array}$ & $\begin{array}{l}\text { incidence of postpartum } \\
\text { hemorrhage (people/\%) }\end{array}$ & $\begin{array}{l}\text { NICU hospitalization } \\
\text { rate (people/\%) }\end{array}$ & $\begin{array}{l}\text { newborns jaundice } \\
\text { (people/\%) }\end{array}$ \\
\hline Before carry out & $6.58 \pm 3.45$ & $24(2.2)$ & $111(10.3)$ & $\begin{array}{l}\text { newborns asphyxia } \\
\text { (people/\%) }\end{array}$ \\
After carry out & $6.54 \pm 3.46$ & $26(2.0)$ & $110(8.6)$ & $9(5.4)$ \\
statistics & 0.335 & 0.117 & 2.086 & $66(5.2)$ \\
$P$ & 0.738 & 0.733 & 0.149 & 0.070 \\
\hline
\end{tabular}

\subsection{Intangible Results}

Before and after the implementation of the activity, the team members' sense of responsibility, application of the quality management circle method, team cohesion, communication and coordination ability, problem solving ability, team sense of achievement, self-confidence and enthusiasm were rated according to a total of 8 indicators, with 5 points being the highest, 3 points being average and 1 point being the lowest. After the scoring is completed, the scores are compared with those before the QCC activities to draw a radar map, as shown in figure 3. According to the statistics, all the index scores of the activity team were improved after the quality control circle activity.

responsibility

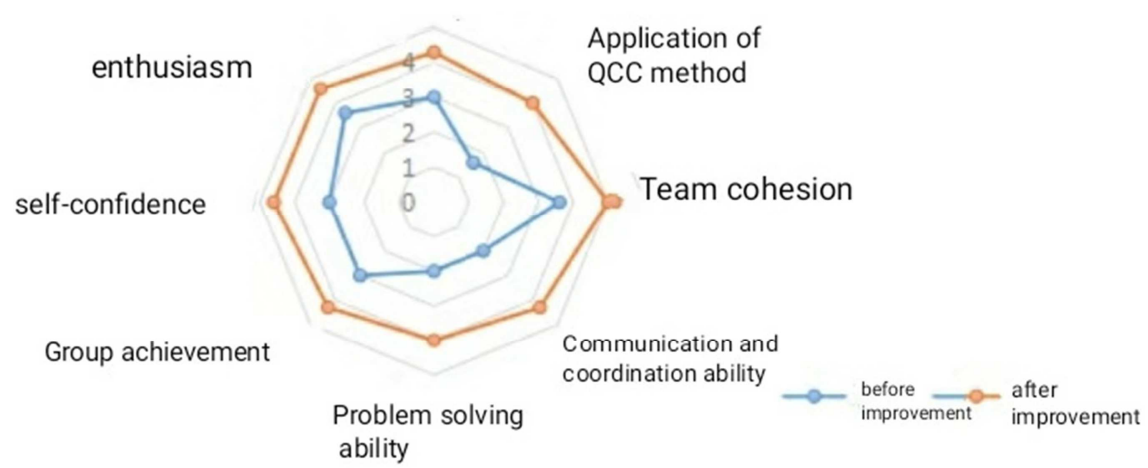

Figure 3. Ability scoring radar chart of group members before and after the implementation of QCC activities. 


\section{Discussion}

\subsection{Quality Control Circle Activities (QCC) Can Improve the Delayed Cord Clamp Rate of Newborns After Vaginal Delivery}

Delayed cord clamp is helpful to stabilize the transition from intrauterine to extrauterine. [11]. However, due to the routine obstetrics, the shortage of obstetric nurse, the donation and preservation of umbilical cord blood and other reasons, [1]. Obstetricians and obstetric nurse did not delayed cord clamp in newborns without contraindications. Through this activity, obstetric nurse identified the indications of delayed cord clamp, formed a standardized process, and implemented it. The results of this study showed that the delayed cord clamp rate of the newborn after vaginal delivery increased from $35.1 \%$ before the activity to $82.3 \%(\mathrm{P}<0.001)$. Meanwhile, there were no statistically significant differences in the duration of the third stage of labor, incidence of postpartum hemorrhage, neonatal hospitalization rate of NICU, incidence of hyperbilirubinemia, and incidence of neonatal asphyxia $(\mathrm{P}>0.05)$. Umbilicus resection immediately after delivery is a routine operation for the third stage of labor to prevent postpartum hemorrhage and placenta retention [1]. However, the results of this study showed that delayed cord clamp resection did not prolong the third stage of labor and increase the risk of postpartum hemorrhage, which is consistent with the results of jialei feng [12] and Andersson [13]. In addition, although delayed cord clamp enabled newborns to obtain additional blood and red blood cells, the results of this study showed that the incidence of neonatal jaundice did not increase, which was consistent with the results of Krueger [14] and fan xuemei [15]. It can be seen that the delay of cord clamp did not increase the incidence of maternal and infant adverse outcomes while increasing neonatal blood volume. The development of the quality control circle activity makes the operation of delayed cord clamp standardized and normalized, and enters the virtuous cycle of continuous improvement, so that more newborns benefit.

\subsection{QCC Activities Enhance the Comprehensive Ability of Obstetric Nurse}

The quality QCC activities improve the cohesion of obstetric nurse' medical teams. The quality service for patients is by no means solely undertaken by the nursing profession. Only by integrating medical care can the "patient-centered" service concept be effective and benefit patients. [16] in this quality control circle activity, our department has carried out the training of integrated medical care, which not only improves the professional knowledge of both sides, but also strengthens the communication and cooperation between the two sides, which enables the two sides to reach an agreement on the implementation of delayed umbilicus cutting for the newborn delivered through vagina, so as to achieve effective cooperation and avoid differences.

Quality management circle activity increased obstetric nurse professional knowledge in this activity, the problem of insufficient knowledge for delay delayed cord clamp, joint department doctor, launched a related study, training, continuing education and training, provide obstetric nurse with professional knowledge growth space, use of learned knowledge and skills, use in the QCC, promote, in order to solve the difficulty of, also help to implement quality management circle activity. [17] standard procedures are also formed to make clinical operations more standardized.

Quality management circle activity increased obstetric nurse circle of self-confidence and enthusiasm QCC activities make each member autonomy, participation, management, under the premise of [18] in team activities, emphasized the importance of individual circle member to the team, encourage them to spontaneous participate in activities, given the opportunity to show, greatly mobilized the enthusiasm of the circle part and make it feel work fun, so as to complete "want me to do" to "I want to make a change."

\section{Conclusion}

The quality control circle activities are fed back and improved through the PDCA cycle to make it more scientific and practical. [19] Through this activity, the delivery room of our hospital identified the indications of delayed cord clamp of newborns after vaginal delivery, and formed a standard process of delayed cord clamp. However, due to the limitations of conditions, our hospital has not yet delayed cord clamp in premature infants and newborns delivered by cesarean section. In future work, we will further search for evidence to explore the application of delayed cord clamp in other types of newborns, so as to bring more benefits to newborns.

\section{References}

[1] Hutchon DJR. Why do obstetricians and obstetric nurse still rush to clamp the cord? [J]. BMJ, 2010 (341): c5447.

[2] Committee on Obstetric Practice. Committee Opinion No. 684: Delayed Umbilical Cord Clamping After Birth [J]. Obstet Gynecol, 2017, 129 (1): e5-e10.

[3] De P C, Herrera J, Garcia C, et al. Effects of delayed cord clamping on the third stage of labour, maternal haematological parameters and acid-base status in fetuses at term [J]. Eur J Obstet Gynecol Reprod Biol, 2016, 207 (9): 153-156.

[4] Mercer J S, Ericksonowens D A, Collins J, et al. Effects of Delayed Cord Clamping on Residual Placental Blood Volume, Hemoglobin and Bilirubin Levels in Term Infants: A Randomized Controlled Trial [J]. Journal of Perinatology, 2017 37 (3): 260-264.

[5] Nevill E, Meyer M P. Effect of delayed cord clamping (DCC) on breathing and transition at birth in very preterm infants $[\mathrm{J}]$. Early Hum Dev, 2015, 91 (7): 407-411.

[6] Brocato B, Holliday N, Jr W R, et al. Delayed Cord Clamping in Preterm newborns: A Review of Benefits and Risks [J]. Obstet Gynecol Surv, 2016, 71 (1): 39-42. 
[7] Liang minghui, liu tingfang, dong siping. Application research of quality control circle in continuous improvement of medical quality [J]. China hospital management, 2012, 32 (2): 37-39.

[8] Hou dongsheng. Carnegie management essence [M]. Beijing: China times economic press, 2002: 393-395.

[9] Zhang xinguo. Practice and skills of hospital quality management circle activities [M]. Hangzhou: zhejiang university press, 2010: 32-34.

[10] Journal of perinatal medicine, 2017, (9): 625-629.

[11] Zhang hui-xin, zhang hong-yu, zhang xiao-li, et al. Effects of delayed umbilicus amputation on neonates $[\mathrm{J}]$. Chinese journal of perinatal medicine, $2014,(10)$ : 716-718.

[12] Feng jialei, liu jun, bao ai-rong, lian ying, wang xue-fei, qi jing, zhai li-hong, han yu-juan. Study on the effect of delayed umbilicus amputation on maternal and infant outcomes in neonates [J]. Chinese journal of nursing, 2008, 53 (02): 144-148.

[13] Andersson, O Hellstrom - Westas L, Dan A, et al. Effects of of compared with early umbilical cord clamping on maternal postpartum hemorrhage and cord blood gas from: A randomized trial $[\mathrm{J}]$. Acta Obstetricia Et Gynecologica Scandinavica, 2013, 92 (5): 567-574.

[14] Krueger M S, Eyal F G, Peevy K J, et al. J of cord clamping with and without cord stripping: A prospective randomized trials of preterm newborns. [J]. American Journal of Obstetrics \& Gynecology reported, 2015, 212 (3): 394. No. e1-394. E5.

[15] Fan xuemei, zhou chunxiu, zhang aixia, li liulan, sun zhiling. Effect of umbilical cord compression on anemia and jaundice in preterm infants with gestational age less than 32 weeks [J]. Chinese journal of nursing, 2008, 53 (02): 149-153.

[16] Li jiping, li li. Exploration of integrated quality service model of medical care $[\mathrm{J}]$. China nursing management, 2012, 12 (12): 5-7.

[17] Zhang feixue, wang lianyue, yu yanyan, li yunxia, xu zhilou, huang jianli. Practice of "quality control circle" activity to improve the comprehensive quality of nursing staff [J]. Chinese modern doctors, 2012, 50 (27): 127-130.

[18] Huang benqing, dong pan, Yang shuxin, lv qiuyan. Problems and Suggestions on the development of quality management circle in clinical nursing [J]. Journal of nursing management, 2015, 15 (03): 227-228.

[19] Fang shu-jing, liu mei, xu qin, xie yu-hua, jin xia, Yin chun-yan, sun liang-ping, jin lei, chang ping. Application of quality control circle in the prevention of lower extremity deep venous thrombosis in patients with bone trauma [J]. Nursing management in China, 2014, 14 (03): 299-301.

[20] Wei Na. Status quo of application of Quality Control Circle in nursing field in China [J]. Chinese General Nursing, 2019, 26: 3246-3248 